\title{
List of Reviewers Contributing to Volume 29, 2020
}

\section{Sibel Erduran ${ }^{1}$}

Accepted: 3 February 2021 / Published online: 10 March 2021

(C) The Author(s), under exclusive licence to Springer Nature B.V. 2021

The Editors of Science \& Education would like to extend their gratitude to the following colleagues who have contributed to the review process of the journal in 2020 by reviewing papers for Volume 29. Their thorough reading and feedback have ensured that the work represented in the journal continues to be subjected to rigorous intellectual scrutiny.

$\begin{array}{lll}\text { Kason Ka Ching Cheung } & \text { Amy Ackerberg-Hastings } & \text { Gustaaf C. Cornelis } \\ \text { Dietmar Hoettecke } & \text { Peter Heering } & \text { TBM Chowdhury } \\ \text { Michael Reiss } & \text { Michael P. Clough } & \text { Salvio Chibeni } \\ \text { Gregory J. Kelly } & \text { Alessandro Zir } & \text { Ying-Chih Chen } \\ \text { Edith Roslyn Dempster } & \text { Huang Xiao } & \text { Tanner Bryan } \\ \text { Yann Benetreau-Dupin } & \text { Allison Witucki } & \text { Richard Brock } \\ \text { William F. McComas } & \text { John Willinsky } & \text { Marc Behrendt } \\ \text { Calvin S. Kalman } & \text { Amy Ricketts } & \text { Ralph M. Barnes } \\ \text { Hagop A. Yacoubian } & \text { Ashlyn Pierson } & \text { Lorenzo Baravalle } \\ \text { Veronica C. } & \text { Wonyong Park } & \text { Andrew Atkinson } \\ \text { McGowan } & \text { Ebru Zeynep Mugaloglu } & \text { Roman Asshoff } \\ \text { Liam Guilfoyle } & \text { Jean-Pierre Marquis } & \text { Sahar Alameh } \\ \text { Colin F. Gauld } & \text { Ricardo Lopes Coelho } & \text { Noa Ageitos } \\ \text { Haira Emanuela Gandolfi } & \text { Robert Kiddell } & \text { Ana Sofia Afonso } \\ \text { Glenn Dolphin } & \text { Rosaria Justi } & \text { Cibelle Celestino Silva } \\ \text { Dana M. Christensen } & \text { Ellen K. Henriksen } & \text { John L Rudolph } \\ \text { Meghan Odsliv Bratkovich } & \text { Karen Haydock } & \text { Kevin McCain } \\ \text { Norman G. Lederman } & \text { Yannis Hadzigeorgiou } & \text { Ismo Koponen } \\ \text { Laura Branchetti } & \text { Judith Lederman } & \text { Ingo Eilks } \\ \text { Pablo Antonio Archila } & \text { Silvia Ferreira } & \text { Agustin Aduriz-Bravo } \\ \text { Zoubeida R. Dagher } & \text { Lynda Dunlop } & \text { Sergio Zappoli } \\ \text { Konstantina Antiochou } & \text { Carmen S Dixon } & \text { Leon Walls } \\ \text { Joseph Agassi } & \text { Yang Deng } & \text { Frederico Augusto Toti } \\ & & \end{array}$

Sibel Erduran

Sibel.Erduran@education.ox.ac.uk

1 Department of Education, University of Oxford, 15 Norham Gardens, Oxford OX2 6PY, UK 
John Tawa

Giulia Tasquier

Vicente Talanquer

Nelson Studart

Michael T. Stuart

Tiffany-Rose Sikorski

Harry L. Shipman

Jane Sheldon

Samuel Molina Schnorr

Kelly Marie Schmid

Jeffrey Daniel Radloff

Gregory Radick

Hans Radder

Paulo Mauricio

Martha Marandino

Rosana L. F. Silva

Olivia Levrini

Matteo Leone

Laurinda Leite

Kostas Kampourakis

Rut Jimenez-Liso

Tetsuji Iseda

Lena Hansson

Jonas Hallstrom

Jesper Haglund

Jenaro Guisasola

Richard Grandy

Rachel Gisewhite

Leticia Garcia-Romano

Antonio Garcia-Carmona

Arthur Galamba

Janice Marie Fulford

Nadja Frerichs

Olival Freire Jr.

M. Fowler

Khadija Engelbrecht Fouad

Veronica S. Flodin

Gabriela Kaiana Ferreira

Claudio Fazio

Ayca K. Fackler

Eugenia Etkina

Ebru Eren

Deborah Dutta

Richard Duschl

Volkan Duran

Brian Matthew Donovan
Maria C.

Domanguez-Sales

Ricardo S. do Carmo

Justin Dillon

Thomas Dillern

Paulo de Carvalho

Saouma BouJaoude

Steven Borish

Paulo de Faria Borges

Sarah B. Boesdorfer

Brandon Boesch

Nelio M.V. Bizzo

Tim C.

Billion-Kramer

Fabio Bevilacqua

Eleonora Barelli

Lucy Avraamidou

Dayle Anderson

Georgios Ampatzidis

Luciana Resende Allain

Milena Raquel Alcocer

Joana Guilares Aguiar

Andreas Stylianides

David W. Rudge

Thomas Reydon

Maria E.B. Prestes

Mauricio Pietrocola

Erik L Peterson

Mansoor Niaz

Ross H. Nehm

Don Metz

Ricardo Karam

Theodore Arabatzis

Erica Zorrilla

G. Cigdem Yalcin

James A. Wilson

Jessica Watkins

Ke Wang

Paul A. Wagner

Liliana Valladares

Celebi Uluyol

Constantinos Tzanakis

Pasquale Tucci

Maria Elena Truyol

Bojan M. Tomic

Amber Todd

Frederike Tirre
Italo Testa

Zenko Takayama

Keith S Taber

Ryan Summers

Molly A.M. Stuhlsatz

Shawn Stevens

Constantina Stefanidou

Dimitris Stavrou

Yilmaz Soysal

Cary Sneider

Carol L. Smith

Xiaoming Shi

Leona Schauble

Jerome Santini

Ramona Rusu

Federica Russo

Ernani V. Rodrigues

Brandon Reynante

Pedro Rocha Reis

Andreas Redfors

Terrance Joseph Quinn

Blanca Puig

Edward E. Prather

Jacob Pleasants

Luis Paulo Piassi

Erin E. Peters-Burton

Osvaldo Pessoa Jr.

Robert T. Pennock

Sharon Pelech

Jorgen Paul

Mitch O'Toole

Jairo Ortiz-Revilla

Noushin Nouri

Alfred Nordmann

Argyris Nipyrakis

Danusa Munford

Bridget K. Mulvey

Francesca Morselli

Victoria Millar

Pavlos Mihas

Hanno Michel

Abdeljalil Metioui

D. Amaral Taveira Mello

Christine V McDonald

William J. McConnell

Isabel P. Martins

Fabio Marineli 


\begin{tabular}{|c|c|c|}
\hline Maria Jose Madrid & Krystallia Halkia & Adam Devitt \\
\hline Juliana Machado & Candice Guy-Gaytain & Hasan Deniz \\
\hline Francois Lombard & David Guillemette & Dayna Jean DeFeo \\
\hline Bruce Lewenstein & Sarah Kathryn Guffey & Henk W. de Regt \\
\hline Ralph Levinson & Hajo Greif & Cecile de Hosson \\
\hline Thomas Mark Lessl & Ileana Maria Greca & Kevin Charles de Berg \\
\hline Eun Ah Lee & Joseph Graves Jr & Alison Cullinane \\
\hline Gulsen Leblebicioglu & Caren A Gough & Saul Alejandro Contreras \\
\hline Philippe Lautesse & Andreas Giransson & Pedro D. Colombo Jr \\
\hline Yvonne Lampert & Leonardo M. G. Galli & Leonardo Colletti \\
\hline Rodrigo Lages Silva & Amanda Glaze & Michael Robert Cohen \\
\hline Adam Laats & Vetti Giri & Pierre Clement \\
\hline Kaarle V. J. Kurki-Suonio & Jinyeong Gim & John Clement \\
\hline Ramjit Kumar & Niklas Markus Gericke & Mei-Hung Chiu \\
\hline Deanna Kuhn & Hugh G. Gauch Jr. & Ka Lok Cheng \\
\hline Jeffrey Kovac & Peter Garik & Hamilton V Chaves \\
\hline Naji Kortam & Leticia Gallegos-Cazares & Robert N. Carson \\
\hline Kostas Korfiatis & Linda Fuselier & Luis Miguel Carolino \\
\hline Christiane Konnemann & Alejandro Fuentes-Penna & Cecilia Caiman \\
\hline Mario Koetter & Karen Francoise & Valeria M. Cabello \\
\hline Soren Harnow Klausen & Samantha Fowler & Jenny Byrne \\
\hline Nahum Kipnis & Erik Fooladi & Stephen R. Burgin \\
\hline Magdalena Kersting & Fernando Flores-Camacho & Nathalie Bulle \\
\hline $\mathrm{Li} \mathrm{Ke}$ & Amy Fisher & Jeanne Brunner \\
\hline Ebru Kaya & Belen Fernandez-Sanchez & James Robert Brown \\
\hline Yilmaz Kara & Carmen Fernandez & Guilherme Brockington \\
\hline Hui Jin & Angelo Fasce & Martin J. Bremer \\
\hline Hunkoog Jho & Amy Voss Farris & Zoe Buck Bracey \\
\hline MerceIzquierdo i Aymerich & Mik Fanguy & Mark Borrello \\
\hline Tetsuo Isozaki & Manfred Euler & Dirk Jan Boerwinkel \\
\hline Damian Islas & Charbel N. El-Hani & George M Bodner \\
\hline Gurol Irzik & Emil Eidin & Nicholas William Best \\
\hline Elaine Howes & Monica Edwards-Schachter & Ugo Besson \\
\hline HayatHokayem & Luiz Henrique Dutra & Eugenio Bertozzi \\
\hline Steven J. Hoekstra & Ravit G. Duncan & Giray Berberoglu \\
\hline Trond Harkjerr & Washington T. Dudu & Federico Benitez \\
\hline Tamara S. Hancock & Thomas Drucker & Alex Bellucco \\
\hline Karim Hamza & Bangping Ding & Richard Bellon \\
\hline Marcus Hammann & Aurel Harrison Diamond & Renata da Fonseca M. E \\
\hline
\end{tabular}


Igor Bascandziev

Berta Barquero

Hartono Bancong

Janelle Bailey

Natalia Helena Azevedo

Zekai Ayik

Elena Ausejo
Amir Asghari

Richard Arthur

Angel Arcos-Vargas

Leonardo A. L. Araujo

Fabiano Antunes

Mayra Antonelli-Ponti

Allison Antink-Meyer
Tamer Amin

G. Donald Allen

Selin Akgun

Valarie Akerson

Orlando Aguiar Jr

Marianne Foss Achiam

Publisher's Note Springer Nature remains neutral with regard to jurisdictional claims in published maps and institutional affiliations. 